\title{
Circular and Periodic Circular Profiles in a Rectangular Cross Section Along the Straight Waveguide
}

\author{
Z. Menachem ${ }^{1} \&$ S. Tapuchi ${ }^{1}$ \\ ${ }^{1}$ Department of Electrical and Electronics Engineering, Sami Shamoon College of Engineering, Israel \\ Correspondence: Z. Menachem, Department of Electrical and Electronics Engineering, Sami Shamoon College of \\ Engineering, Israel. E-mail: zionm@post.tau.ac.il
}

\author{
Received: February 15, 2015 Accepted: March 1, 2015 Online Published: March 20, 2015 \\ doi:10.5539/apr.v7n2p121 URL: http://dx.doi.org/10.5539/apr.v7n2p121
}

\begin{abstract}
The first objective of this paper is to present a technique and a particular application to calculate the dielectric profile, the elements of the matrix, and its derivatives of the dielectric profile in the cases of circular and periodic circular profiles in the cross section of the rectangular straight waveguide. The second objective is to investigate the influence of the circular and periodic circular profiles on the output fields. The proposed technique relates to the method that is based on the Laplace and Fourier transforms, the inverse Laplace and Fourier transforms. This model is useful to predict the structure of the output fields for circular and periodic circular profiles in a rectangular metallic waveguide. The application is useful for straight waveguides in the microwave and the millimeter-wave regimes.
\end{abstract}

Keywords: wave propagation, dielectric profiles, straight metallic waveguide, rectangular cross section, dielectric waveguide

\section{Introduction}

The methods of straight waveguides have been proposed in the literature. A method of selective suppression of electromagnetic modes in rectangular waveguides by loading distributed losses in some special position of waveguide inner wall has been presented (Jiao, 2011). In this paper, the unwanted modes can be attenuated much larger relative to the operating mode and this proposed method can be used to improve the stability of rectangular waveguide beam-wave interaction circuit. An analytical model for the corrugated rectangular waveguide has been extended to compute the dispersion and interaction impedance (Mineo, 2010). In this paper, the application of analytical method based on the field equations, validated by 3-D simulators has been presented to design corrugated rectangular waveguide slow-wave structure $T H_{z}$ amplifiers.

A fundamental and accurate technique to compute the propagation constant of waves in a lossy rectangular waveguide has been proposed (Yeap et al., 2011). This method is based on matching the electric and magnetic fields at the boundary, and allowing the wavenumbers to take complex values. An important consequence of this work is the demonstration that the loss computed for degenerate modes propagating simultaneously is not simply additive.

A method of solving the propagation constant for the bound modes in the dielectric rectangular waveguides has been presented (Sharma, 2010). In this paper, the characteristic equations of the modes of the dielectric rectangular waveguide have been derived using the mode matching technique. A simple closed form expression to compute the time-domain reflection coefficient for a transient $T E_{10}$ mode wave incident on a dielectric step discontinuity in a rectangular waveguide has been presented (Rothwell et al., 2009). In this paper, an exponential series approximation was provided for efficient computation of the reflected and transmitted field waveforms. The electromagnetic fields in rectangular conducting waveguides filled with uniaxial anisotropic media has been characterized (Liu et al., 2000). In this paper, the electric type dyadic Green's function due to an electric source was derived by using eigenfunctions expansion and the Ohm-Rayleigh medhod. 
The simulation, design and implementation of bandpass filters in rectangular waveguides has been proposed (Choocadee et al., 2012). In this paper, the filters were simulated and designed by using a numerical analysis program based on the wave iterative method. The waveguide filter design simulation allows us to reduce the design problem to determinate a bandpass filter structure in the rectangular waveguide with usable frequency range. A full-vectorial boundary integral equation method for computing guided modes of optical waveguides has been proposed (Lu et al., 2012). This method is applicable to waveguides with high intex-contrast, sharp corners and layered background. The integral equations are used to compute the Neumann-to-Dirichlet operators for sub-domains of constant refractive index on the transverse plane of the waveguide, and they are discretized by a Nystrom method with a graded mesh for handling the corners.

A general method with the equations for the propagation constants of fiber waveguides of arbitrary cross-sectional shapes has been propsed (Eyges et al., 1979). In this paper, the proposed techniques used to solve problem of scattering by irregularly shaped dielectric bodies, and in the static limit, for solving the problem of an irregular dielectric or permeable body in an external field. The phase of the reflection coefficient of a $T E_{10}$ rectangular waveguide mode at the cut-off point in a gentle downtaper has been investigated (Soekmadji et al., 2009).

The rectangular dielectric waveguide technique for the determination of complex permittivity of a wide class of dielectric materials of various thicknesses and cross sections has been described (Abbas et al., 1998). In this paper, the technique has been presented to determine the dielectric constant of materials. By transforming the infinite $x-y$ plane onto a unit square and using two-dimensional Fourier series expansions, the propagation constants and modal fields of dielectric waveguides have been accurately calculated (Hewlett et al., 1995). This method is reliable down to modal cutoff and can be used to determine cutoff V-values directly.

A transfer matrix function for the analysis of electromagnetic wave propagation along the straight dielectric waveguide with arbitrary profiles has been proposed (Menachem \& Jerby, 1998). This method based on the Laplace and Fourier transforms. This method is based on Fourier coefficients of the transverse dielectric profile and those of the input wave profile. Laplace transform is necessary to obtain the comfortable and simple input-output connections of the fields. The transverse field profiles are computed by the inverse Laplace and Fourier transforms.

All models that are mentioned refer to solve interesting wave propagation problems with a particular geometry. If we want to solve more complex problems of coatings in the cross-section of the dielectric waveguides, such as periodic profiles in the cross section, then it is important to develop in each modal an improved technique for calculating profiles with the dielectric coatings or with the periodic structure.

This paper presents a technique and a particular application to calculate the dielectric profile, the elements of the matrix and its derivatives of the dielectric profile in the cases of circular and periodic circular profiles in the cross section of the straight rectangular waveguide. The examples will be demonstrated for the circular and periodic circular dielectric profiles in a rectangular metallic waveguide. The proposed technique relates to the method for the propagation along the straight rectangular metallic waveguide (Menachem \& Jerby, 1998). The technique and the particular application to solve the circular and periodic circular profiles in the cross section will given in detail. This model is useful to predict the output results of the fields in the cases of circular and periodic circular profiles in the cross section of the straight rectangular waveguide in the cases of wave millimeter.

\section{Formulation of the Problem}

The objective of this paper is to introduce a technique and a particular application to calculate the dielectric profile, the elements of the matrix and its derivatives of the dielectric profile in the cases of circular and periodic circular profiles in the cross section of the straight rectangular waveguide. 


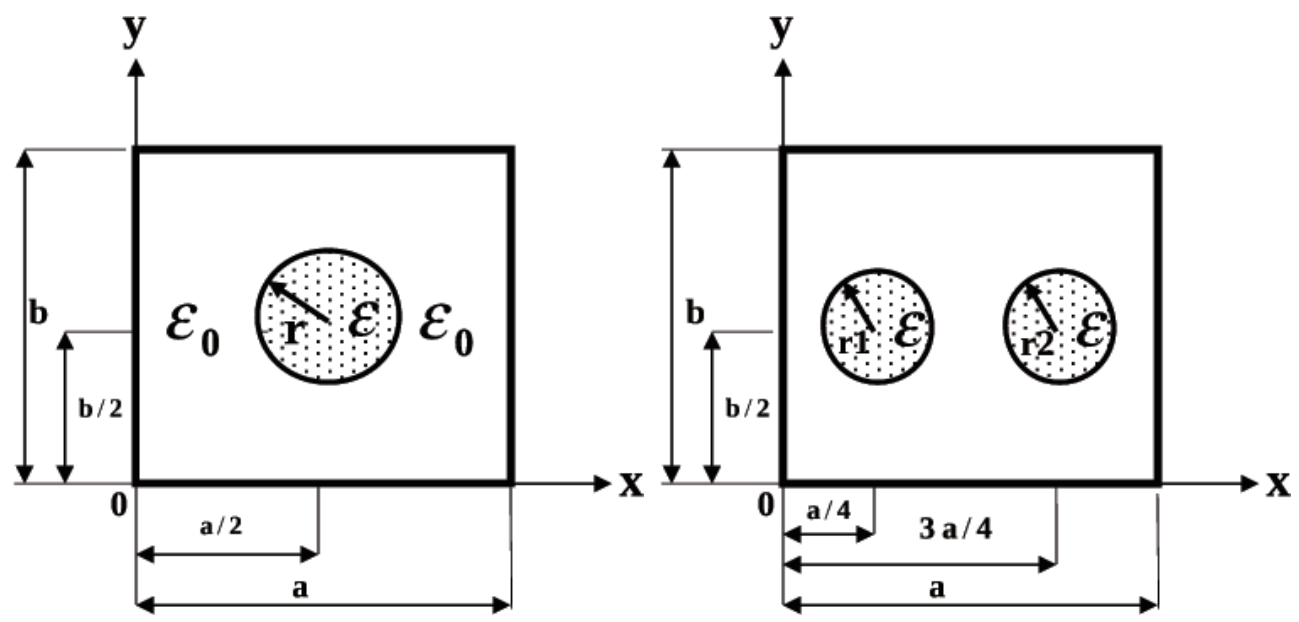

(a)

(b)

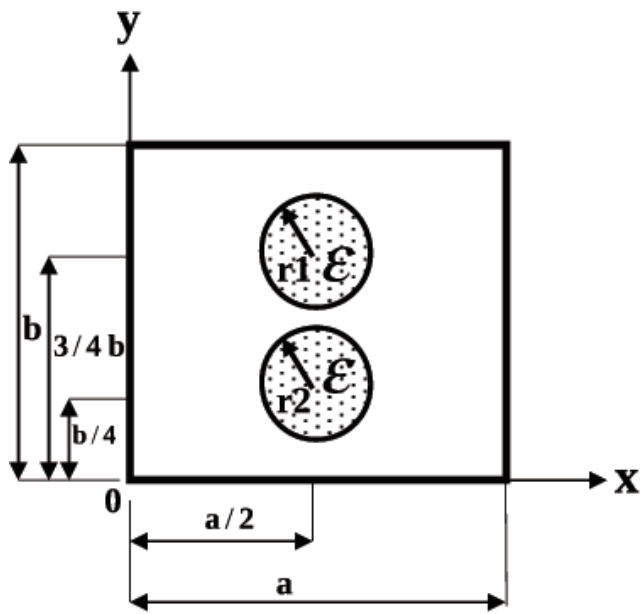

(c)

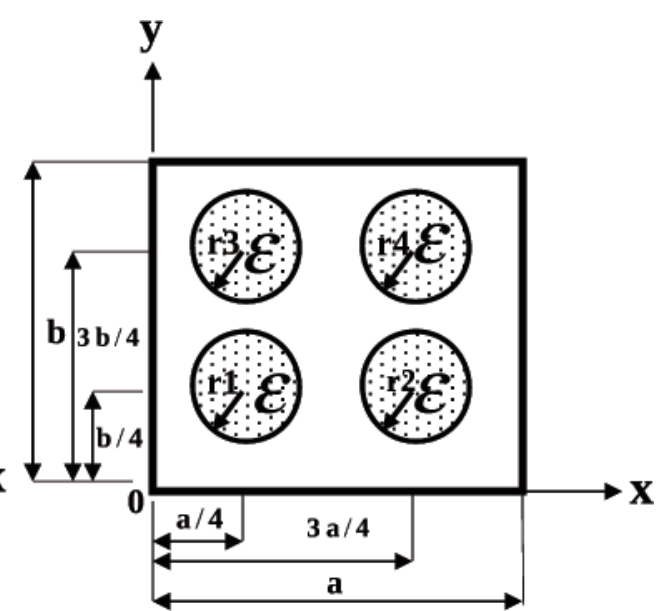

(d)

Figure 1. The circular profiles in the cross section of the straight waveguide (a) One circular profile. (b) Periodic structure with two circular profiles along x-axis. (c) Periodic structure with two circular profiles along y-axis.

(d) Periodic structure with four circular profiles along $\mathrm{x}$-axis and $\mathrm{y}$-axis

Four examples of circular and periodic circular profiles in the cross section of the straight waveguide are shown in Figures 1(a)-(d). Figure 1(a) shows one circular profile where the center is located at the point (a/2, b/2). Figure 1(b) shows an example of periodic structure with two circular profiles along $x$-axis. In this example, the center of the left circle is located at the point $(\mathrm{a} / 4, \mathrm{~b} / 2)$ and the center of the right circle is located at the point (3/4 a, b/2).

Figure 1(c) shows an example of periodic structure with two circular profiles along y-axis. In this example, the center of the upper circle is located at the point $(\mathrm{a} / 2,3 / 4 \mathrm{~b})$ and the center of the lower circle is located at the point $(\mathrm{a} / 2, \mathrm{~b} / 4)$. Figure 1(d) shows an example of periodic structure with four circular profiles along $\mathrm{x}$-axis and y-axis. In this example, the center of the first circle is located at the point $(\mathrm{a} / 4, \mathrm{~b} / 4)$, the center of the second circle is located at the point $(3 / 4 \mathrm{a}, \mathrm{b} / 4)$, the center of the third circle is located at the point $(\mathrm{a} / 4 \mathrm{a}, 3 / 4 \mathrm{~b})$, and the center of the firth circle is located at the point $(3 / 4 \mathrm{a}, 3 / 4 \mathrm{~b})$.

The objective of this paper is to introduce a technique and a particular application to calculate the dielectric profile, the elements of the matrix and its derivatives of the dielectric profile in the cases of circular and periodic circular profiles (Figures 1(a)-(d)) in the cross section of the straight rectangular waveguide. The proposed technique relates to the method for the propagation along a straight rectangular metallic waveguide (Menachem \& Jerby, 1998). The method is based on the Laplace and Fourier transforms, the inverse Laplace and Fourier transforms. Laplace transform is necessary to obtain the comfortable and simple input-output connections of the fields. The output transverse field profiles are computed by the inverse Laplace and Fourier transforms. The output components of 
the electric field are given finally by

$$
\begin{gathered}
\mathbf{E}_{\mathbf{x}}=\left\{\mathbf{D}_{\mathbf{x}}+\alpha_{1} \mathbf{M}_{\mathbf{1}} \mathbf{M}_{2}\right\}^{-1}\left(\hat{\mathbf{E}}_{\mathbf{x}_{0}}-\alpha_{2} \mathbf{M}_{\mathbf{1}} \hat{\mathbf{E}}_{\mathbf{y}_{\mathbf{0}}}\right), \\
\mathbf{E}_{\mathbf{y}}=\left\{\mathbf{D}_{\mathbf{y}}+\alpha_{\mathbf{1}} \mathbf{M}_{\mathbf{3}} \mathbf{M}_{\mathbf{4}}\right\}^{-\mathbf{1}}\left(\hat{\mathbf{E}}_{\mathbf{y}_{0}}-\alpha_{\mathbf{3}} \mathbf{M}_{\mathbf{3}} \hat{\mathbf{E}}_{\mathbf{x}_{0}}\right), \\
\mathbf{E}_{\mathbf{z}}=\mathbf{D}_{\mathbf{z}}^{-\mathbf{1}}\left\{\hat{\mathbf{E}}_{\mathbf{z}_{0}}+\frac{\mathbf{1}}{\mathbf{2 s}}\left(\mathbf{G}_{\mathbf{x}} \mathbf{E}_{\mathbf{x}_{\mathbf{0}}}+\mathbf{G}_{\mathbf{y}} \mathbf{E}_{\mathbf{y}_{\mathbf{0}}}\right)-\frac{\mathbf{1}}{\mathbf{2}}\left(\mathbf{G}_{\mathbf{x}} \mathbf{E}_{\mathbf{x}}+\mathbf{G}_{\mathbf{y}} \mathbf{E}_{\mathbf{y}}\right)\right\},
\end{gathered}
$$

where $\mathbf{E}_{\mathbf{x}_{0}}, \mathbf{E}_{\mathbf{y}_{0}}, \mathbf{E}_{\mathbf{z}_{0}}$ are the initial values of the corresponding fields at $\mathrm{z}=0$, i.e., $\mathbf{E}_{\mathbf{x}_{0}}=\mathbf{E}_{\mathbf{x}}(\mathrm{x}, \mathrm{y}, \mathrm{z}=0)$, and $\hat{\mathbf{E}}_{\mathbf{x}_{0}}, \hat{\mathbf{E}}_{\mathbf{y}_{0}}, \hat{\mathbf{E}}_{\mathbf{z}_{0}}$ are the initial-value vectors.

The modified wave-number matrices are given by

$$
\mathbf{D}_{\mathbf{x}} \equiv \mathbf{K}^{(\mathbf{0})}+\frac{k_{o}^{2} \chi_{0}}{2 s} \mathbf{G}+\frac{j k_{o x}}{2 s} \mathbf{N G}_{\mathbf{x}}, \quad \mathbf{D}_{\mathbf{y}} \equiv \mathbf{K}^{(\mathbf{0})}+\frac{k_{o}^{2} \chi_{0}}{2 s} \mathbf{G}+\frac{j k_{o y}}{2 s} \mathbf{M G}_{\mathbf{y}}, \quad \mathbf{D}_{\mathbf{z}} \equiv \mathbf{K}^{(\mathbf{0})}+\frac{k_{o}^{2} \chi_{0}}{2 s} \mathbf{G},
$$

where the diagonal matrices $\mathbf{K}^{(\mathbf{0})}, \mathbf{M}$, and $\mathbf{N}$ are given by

$$
\begin{gathered}
\left(\mathbf{K}^{(0)}\right)_{(\mathrm{n}, \mathrm{m})\left(\mathrm{n}^{\prime}, \mathrm{m}^{\prime}\right)}=\left\{\left[\mathbf{k}_{o}^{2}-(\mathrm{n} \pi / \mathrm{a})^{2}-(\mathrm{m} \pi / \mathrm{b})^{2}+\mathrm{s}^{2}\right] / 2 \mathrm{~s}\right\} \delta_{n n^{\prime}} \delta_{m m^{\prime}}, \\
\mathbf{M}_{(\mathrm{n}, \mathrm{m})\left(\mathrm{n}^{\prime}, \mathrm{m}^{\prime}\right)}=\mathrm{m} \delta_{\mathrm{nn}^{\prime}} \delta_{\mathrm{mm}^{\prime}}, \quad \mathbf{N}_{(\mathrm{n}, \mathrm{m})\left(\mathrm{n}^{\prime}, \mathrm{m}^{\prime}\right)}=\mathrm{n} \delta_{\mathrm{nn}^{\prime}} \delta_{\mathrm{mm}^{\prime}},
\end{gathered}
$$

and where

$$
\begin{gathered}
\alpha_{1}=\frac{k_{o x} k_{o y}}{4 s^{2}}, \quad \alpha_{2}=\frac{j k_{o x}}{2 s}, \quad \alpha_{3}=\frac{j k_{o y}}{2 s}, \\
\mathbf{M}_{\mathbf{1}}=\mathbf{N G}_{\mathbf{y}} \mathbf{D}_{\mathbf{y}}^{-1}, \quad \mathbf{M}_{\mathbf{2}}=\mathbf{M G}_{\mathbf{x}}, \quad \mathbf{M}_{\mathbf{3}}=\mathbf{M G}_{\mathbf{x}} \mathbf{D}_{\mathbf{x}}^{-1}, \quad \mathbf{M}_{\mathbf{4}}=\mathbf{N G}_{\mathbf{y}} .
\end{gathered}
$$

Similarly, the other components of the magnetic field are obtained. The output transverse field profiles are given by the inverse Laplace and Fourier transforms, as follows

$$
\mathbf{E}_{\mathbf{y}}(\mathrm{x}, \mathrm{y}, \mathrm{z})=\sum_{n} \sum_{m} \int_{\sigma-j_{\infty}}^{\sigma+j_{\infty}} \mathbf{E}_{\mathbf{y}}(\mathrm{n}, \mathrm{m}, \mathrm{s}) \exp \left[\mathrm{jnk}_{\mathrm{ox}} \mathrm{x}+\mathrm{jmk}_{\mathrm{oy}} \mathrm{y}+\mathrm{sz}\right] \mathrm{ds},
$$

where the inverse Laplace transforms is calculated according to the Salzer method (Salzer, 1955) (Salzer, 1961). A Fortran code is developed using NAG subroutines (The Numerical Algorithms Group (NAG)).

It is very interesting to compare between the mode model method for wave propagation in the straight waveguide with a circular cross section (Menachem \& Tapuchi, 2013) and this model for the wave propagation in the straight waveguide with a rectangular cross section. These are two kinds of different methods that enable us to solve practical problems with different boundary conditions. The calculations in all method are based on using Laplace and Fourier transforms, and the output fields are computed by the inverse Laplace and Fourier transforms. Laplace transform on the differential wave equations is needed to obtain the wave equations (and thus also the output fields) that are expressed directly as functions of the transmitted fields at the entrance of the waveguide at $z=0^{+}$. Thus, the Laplace transform is necessary to obtain the comfortable and simple input-output connections of the fields.

The technique and the particular application are proposed in the next section to calculate the dielectric profile $g(x, y)$ and the derivatives $g_{x}(x, y), g_{y}(x, y)$ according to $g_{x}(x, y)=(1 / \epsilon(x, y))(d \epsilon(x, y) / d x), \epsilon(x, y)=\epsilon_{0}(1+g(x, y))$ , and the elements of the matrix $g(n, m)$, and its derivatives $g_{x}(n, m)$ and $g_{y}(n, m)$. The examples and the graphical results are given in the cases of circular and periodic circular profiles (Figures 1(a)-(d)) in the cross section of the straight rectangular waveguide.

\section{The Technique and the Particular Application}

The technique, the particular application and several examples are demonstrated in this section in order to calculate the dielectric profile and the derivatives of the dielectric profile in the cases of circular and periodic circular profiles, as shown in Figures 1(a)-(d). 
In this section, we will introduce the dielectric profile $g(x, y)$, the derivatives $g_{x}(x, y), g_{y}(x, y)$, the elements of the matrix $g(n, m)$, and its derivatives $g_{x}(n, m)$ and $g_{y}(n, m)$.

The output results are demonstrated for one circular profile (Figure 1(a)) and for periodic circular profiles (Figures 1(b)-(d)) in the cross section of the straight rectangular waveguide.

\subsection{The Technique}

The elements of the matrix $\mathrm{g}(\mathrm{n}, \mathrm{m})$ are calculated for an arbitrary profile in the cross section of the straight waveguide according to Figure 2.

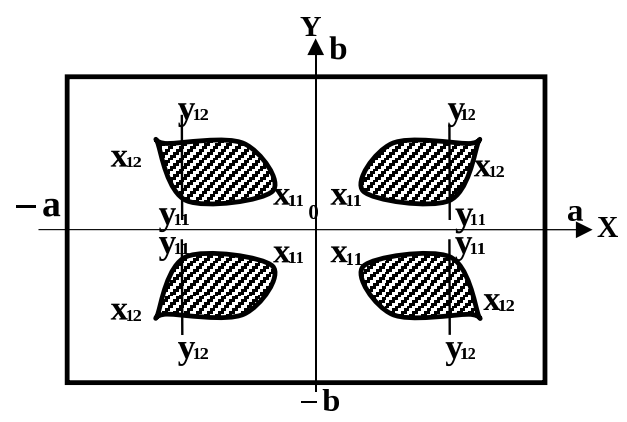

Figure 2 . The arbitrary profile in the cross section

The dielectric profile $g(x, y)$ is given according to $\epsilon(x, y)=\epsilon_{0}(1+g(x, y))$. According to Figure 2 and for $g(x, y)=g_{0}$, we obtain

$$
\begin{gathered}
g(n, m)=\frac{g_{0}}{4 a b} \int_{-a}^{a} d x \int_{-b}^{b} \exp \left[-j\left(k_{x} x+k_{y} y\right)\right] d y \\
=\frac{g_{0}}{4 a b}\left\{\int_{x_{11}}^{x_{12}} d x \int_{y_{11}}^{y_{12}} \exp \left[-j\left(k_{x} x+k_{y} y\right)\right] d y+\int_{-x_{12}}^{-x_{11}} d x \int_{y_{11}}^{y_{12}} \exp \left[-j\left(k_{x} x+k_{y} y\right)\right] d y\right. \\
\left.+\int_{-x_{12}}^{-x_{11}} d x \int_{-y_{12}}^{-y_{11}} \exp \left[-j\left(k_{x} x+k_{y} y\right)\right] d y+\int_{x_{11}}^{x_{12}} d x \int_{-y_{12}}^{-y_{11}} \exp \left[-j\left(k_{x} x+k_{y} y\right)\right] d y\right\} .
\end{gathered}
$$

The development of Equation 3 is given in detail in the Appendix A, and if $y_{11}$ and $y_{12}$ are not functions of $\mathrm{x}$, then the dielectric profile is given by

$$
g(n, m)=\frac{g_{0}}{a b} \int_{x_{11}}^{x_{12}} \cos \left(k_{x} x\right) d x \int_{y_{11}}^{y_{12}} \cos \left(k_{y} y\right) d y .
$$

If $y_{11}$ and $y_{12}$ are functions of $\mathrm{x}$, then we obtain

$$
\begin{gathered}
g(n, m)=\frac{g_{0}}{a b k_{y}} \int_{x_{11}}^{x_{12}}\left[\sin \left(k_{y} y_{12}\right)-\sin \left(k_{y} y_{11}\right)\right] \cos \left(k_{x} x\right) d x \\
=\frac{2 g_{0}}{a m \pi} \int_{x_{11}}^{x_{12}} \sin \left[\frac{k_{y}}{2}\left(y_{12}-y_{11}\right)\right] \cos \left[\frac{k_{y}}{2}\left(y_{12}+y_{11}\right)\right] \cos \left(k_{x} x\right) d x,
\end{gathered}
$$

where the form of the matrix $\mathbf{G}$ is given by:

$$
\mathbf{G}=\left[\begin{array}{cccclcc}
g_{00} & g_{-10} & g_{-20} & \ldots & g_{-n m} & \ldots & g_{-N M} \\
g_{10} & g_{00} & g_{-10} & \ldots & g_{-(n-1) m} & \ldots & g_{-(N-1) M} \\
g_{20} & g_{10} & \ddots & \ddots & \ddots & & \\
\vdots & g_{20} & \ddots & \ddots & \ddots & & \\
g_{n m} & \ddots & \ddots & \ddots & g_{00} & \vdots & \\
\vdots & & & & & & \\
g_{N M} & \ldots & \ldots & \ldots & \ldots & \ldots & g_{00}
\end{array}\right] .
$$


The derivative of the dielectric profile in the case of $y_{11}$ and $y_{12}$ are functions of $\mathrm{x}$, is given by

$$
g_{x}(n, m)=\frac{2}{a m \pi} \int_{x_{11}}^{x_{12}} g_{x}(x, y) \sin \left[\frac{k_{y}}{2}\left(y_{12}-y_{11}\right)\right] \cos \left[\frac{k_{y}}{2}\left(y_{12}+y_{11}\right)\right] \cos \left(k_{x} x\right) d x,
$$

where $g_{x}(x, y)=(1 / \epsilon(x, y))(d \epsilon(x, y) / d x), \epsilon(x, y)=\epsilon_{0}(1+g(x, y)), k_{x}=(n \pi x) / a$, and $k_{y}=(m \pi y) / b$. Similarly, we can calculate the value of $g_{x}(n, m)$, where $g_{y}(x, y)=(1 / \epsilon(x, y))(d \epsilon(x, y) / d y)$.

The equation for one circle is given by $(x-a / 2)^{2}+(y-b / 2)^{2}=r^{2}$, where the center of the circle is located at $(\mathrm{a} / 2, \mathrm{~b} / 2)$, as shown in Figure 1(a). Thus we obtain two possibilities $y_{11}(x)=b / 2-\sqrt{r^{2}-(x-a / 2)^{2}}$ and $y_{12}(x)=b / 2+\sqrt{r^{2}-(x-a / 2)^{2}}$, according to Figure 2. In this case, we obtain $y_{12}-y_{11}=2 \sqrt{r^{2}-(x-a / 2)^{2}}$ and $y_{12}+y_{11}=b$.

In the same principle we can calculate the equations for the periodic circles according to their location, as shown in Figures 1(b)-(d).

\subsection{The particular application based on $\omega_{\varepsilon}$ function}

The particular application is based on the $\omega_{\varepsilon}$ function (Vladimirov, 1971). The $\omega_{\varepsilon}$ function is used in order to solve circular profile and periodic circular profiles in the cross section of the straight waveguide. The $\omega_{\varepsilon}$ function (Figure $3(\mathrm{a}))$ is defined as

$$
\omega_{\varepsilon}(r)= \begin{cases}C_{\varepsilon} e^{-\frac{\varepsilon^{2}}{\varepsilon^{2}-|r|^{2}}} & |r| \leq \varepsilon \\ 0 & |r|>\varepsilon\end{cases}
$$

where $C_{\varepsilon}$ is a constant, and $\int \omega_{\varepsilon}(r) d r=1$.

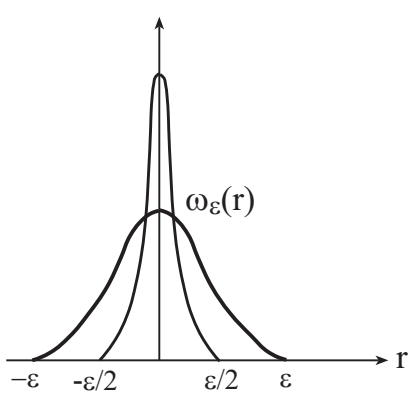

(a)

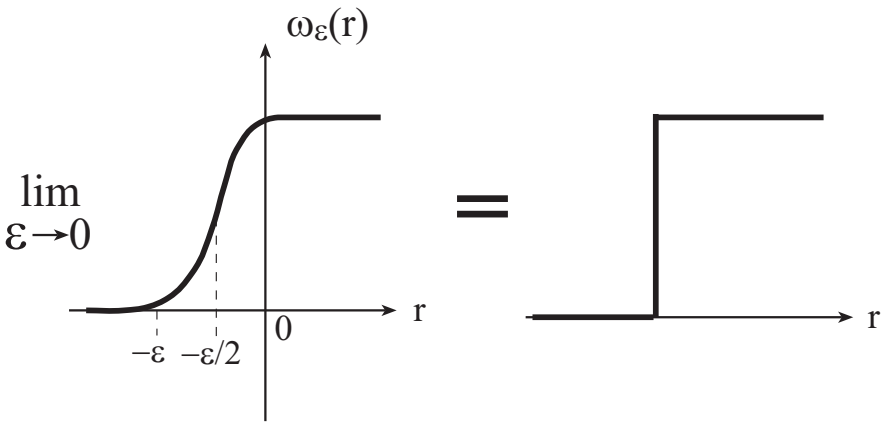

(b)

Figure 3. (a) The $\omega_{\varepsilon}$ function. (b) The $\omega_{\varepsilon}$ function in the limit $\varepsilon \longrightarrow 0$

In the limit $\varepsilon \longrightarrow 0$, the $\omega_{\varepsilon}$ function (Equation 8 ) is shown in Figure 3(b). The dielectric profile for one circle is given where the center is located at $(\mathrm{a} / 2, \mathrm{~b} / 2)$ (Figure $1(\mathrm{a}))$ by

$$
g(x, y)=\left\{\begin{array}{ll}
g_{0} & 0 \leq r<r_{1}-\varepsilon_{1} / 2 \\
g_{0} \exp \left[1-q_{\epsilon}(r)\right] & r_{1}-\varepsilon_{1} / 2 \leq r<r_{1}+\varepsilon_{1} / 2
\end{array},\right.
$$

where

$$
q_{\epsilon}(r)=\frac{\varepsilon_{1}^{2}}{\varepsilon_{1}^{2}-\left[r-\left(r_{1}-\varepsilon_{1} / 2\right)\right]^{2}},
$$

else $g(x, y)=0$. The radius of the circle is given by $r=\sqrt{(x-a / 2)^{2}+(y-b / 2)^{2}}$. Similarly, we can calculate the circular dielectric profile according to their location.

Thus, the derivatives of the dielectric profile for one circle are given where the center is located at (a/2, b/2) (Figure 1(a)) in the region $r_{1}-\varepsilon_{1} / 2 \leq r<r_{1}+\varepsilon_{1} / 2$ by 


$$
\begin{gathered}
g_{x}=\frac{-2 g_{0} \cos \theta \exp \left[1-q_{\epsilon}(r)\right]\left[r-\left(r_{1}-\varepsilon_{1} / 2\right)\right] \varepsilon_{1}{ }^{2}}{\left\{1+g_{0} \exp \left[1-q_{\epsilon}(r)\right]\right\}\left[\varepsilon_{1}{ }^{2}-\left[r-\left(r_{1}-\varepsilon_{1} / 2\right)\right]^{2}\right]^{2}}, \\
g_{y}=\frac{-2 g_{0} \sin \theta \exp \left[1-q_{\epsilon}(r)\right]\left[r-\left(r_{1}-\varepsilon_{1} / 2\right)\right] \varepsilon_{1}{ }^{2}}{\left\{1+g_{0} \exp \left[1-q_{\epsilon}(r)\right]\right\}\left[\varepsilon_{1}{ }^{2}-\left[r-\left(r_{1}-\varepsilon_{1} / 2\right)\right]^{2}\right]^{2}},
\end{gathered}
$$

else $g_{x}=0$, and $g_{y}=0$.

The elements of the matrices for one circle are given where the center is located at $(\mathrm{a} / 2, \mathrm{~b} / 2)$ (Figure 1(a)) by

$$
\begin{gathered}
g(n, m)=\frac{g_{0}}{a b}\left\{\int_{0}^{2 \pi} \int_{0}^{r_{1}-\epsilon_{1} / 2} \cos \left[\frac{n \pi}{a}\left(r \cos \theta+\frac{a}{2}\right)\right] \cos \left[\frac{m \pi}{b}\left(r \sin \theta+\frac{b}{2}\right)\right]+\right. \\
\left.\int_{0}^{2 \pi} \int_{r_{1}-\epsilon_{1} / 2}^{r_{1}+\epsilon_{1} / 2} \cos \left[\frac{n \pi}{a}\left(r \cos \theta+\frac{a}{2}\right)\right] \cos \left[\frac{m \pi}{b}\left(r \sin \theta+\frac{b}{2}\right)\right] \exp \left[1-q_{\epsilon}(r)\right]\right\} r d r d \theta, \\
g_{x}(n, m)=-\frac{2 g_{0}}{a b}\left\{\int_{0}^{2 \pi} \int_{r_{1}-\epsilon_{1} / 2}^{r_{1}+\epsilon_{1} / 2} \frac{\epsilon_{1}^{2}\left[r-\left(r_{1}-\epsilon_{1} / 2\right)\right] \exp \left[1-q_{\epsilon}(r)\right] \cos \theta}{\left[\epsilon_{1}^{2}-\left[r-\left(r_{1}-\epsilon_{1} / 2\right)\right]^{2}\right]^{2}\left[1+g_{0} \exp \left[1-q_{\epsilon}(r)\right]\right]}\right. \\
\left.\cos \left[\frac{n \pi}{a}\left(r \cos \theta+\frac{a}{2}\right)\right] \cos \left[\frac{m \pi}{b}\left(r \sin \theta+\frac{b}{2}\right)\right]\right\} r d r d \theta, \\
g_{y}(n, m)=-\frac{2 g_{0}}{a b}\left\{\int_{0}^{2 \pi} \int_{r_{1}-\epsilon_{1} / 2}^{r_{1}+\epsilon_{1} / 2} \frac{\epsilon_{1}^{2}\left[r-\left(r_{1}-\epsilon_{1} / 2\right)\right] \exp \left[1-q_{\epsilon}(r)\right] \sin \theta}{\left[\epsilon_{1}^{2}-\left[r-\left(r_{1}-\epsilon_{1} / 2\right)\right]^{2}\right]^{2}\left[1+g_{0} \exp \left[1-q_{\epsilon}(r)\right]\right]}\right. \\
\left.\cos \left[\frac{n \pi}{a}\left(r \cos \theta+\frac{a}{2}\right)\right] \cos \left[\frac{m \pi}{b}\left(r \sin \theta+\frac{b}{2}\right)\right]\right\} r d r d \theta,
\end{gathered}
$$

where $r=\sqrt{(x-a / 2)^{2}+(y-b / 2)^{2}}$.

Similarly, we can calculate the periodic circular profile according to the number of the circles and the locations of their centers.

\section{Numerical Results}

The analytical method for the dielectric slab (Collin, 1996) is shown in Figure 4(a). The slab profile in the cross section is based on transcendental equation, as follows

$$
\begin{gathered}
E_{y 1}=j \frac{k_{z}}{\epsilon_{0}} \sin (v x) \quad 0<x<t \\
E_{y 2}=j \frac{k_{z}}{\epsilon_{0}} \frac{\sin (v t)}{\cos (\mu(t-a / 2))} \cos [\mu(x-a / 2)] \quad t<x<t+d \\
E_{y 3}=j \frac{k_{z}}{\epsilon_{0}} \sin [v(a-x)] \quad t+d<x<a,
\end{gathered}
$$

where $v \equiv \sqrt{k_{o}^{2}-k_{z}^{2}}$ and $\mu \equiv \sqrt{\epsilon_{r} k_{o}^{2}-k_{z}^{2}}$ result from the transcendental equation

$$
\left(\frac{a-d}{d}\right) \frac{d \mu}{2} \tan \left(\frac{d \mu}{2}\right)-(t v) \cot (t v)=0 .
$$


The criterion for the convergence of the solution is given by

$$
C(N) \equiv \log \left\{\frac{\max \left(\left|E_{y}^{N+2}-E_{y}^{N}\right|\right)}{\left|\max \left(E_{y}^{N+2}\right)-\min \left(E_{y}^{N}\right)\right|}\right\}
$$

for $N \geq 1$, where $(2 N+1)^{2}$ is the number of the modes. By increasing the order $N$, then $E_{y}(N)$ approaches $E_{y}$.

For the practical case of the straight waveguide with the symmetrical slab profile (Figure 4(a)), Equations (13(a)(c)) are substituted to Equation 1(b) at $z=0^{+}$. The result of the comparison between the theoretical model with the analytical solution (Collin R. E., 1996) is shown in Figure 4(b), where $a=20 \mathrm{~mm}, b=10 \mathrm{~mm}, \mathrm{~d}=3.3 \mathrm{~mm}, \mathrm{t}=8.35$ $\mathrm{mm}, \epsilon_{r}=9$, and $\lambda=6.9 \mathrm{~cm}$. The convergence according to the criterion (14) is shown in Figure 4(c). The value of the criterion between $\mathrm{N}=7$ and $\mathrm{N}=9$ is equal to -2 . Thus, the comparison has shown good agreement.

We have two ways to compare between the results of our model and the other methods.

\subsection{The First Way}

The first way is to compare between the results of the output fields for every order $(\mathrm{N}=1,3,5,7$, and 9$)$ with the final solution of the known method.

\subsection{The Scecond Way}

The second way is to compare between the results of the output fields (according to our model) for every two orders $(\mathrm{N}=1,3, \mathrm{~N}=3,5, \mathrm{~N}=5,7$, and $\mathrm{N}=7,9)$, until our numerical solution is well converged. This way is efficient in the cases that we have complicated problems that we cannot compare with the other final known method.

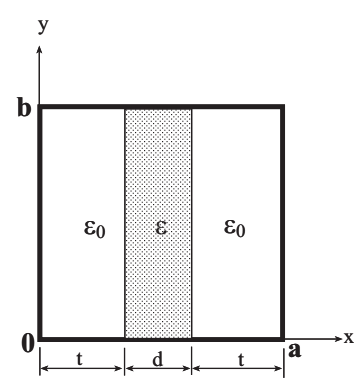

(a)

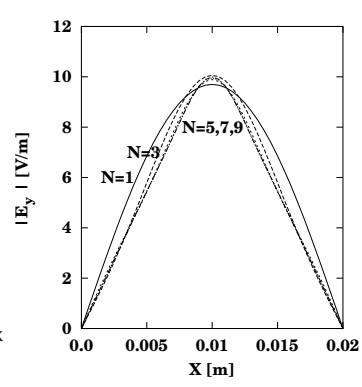

(b)

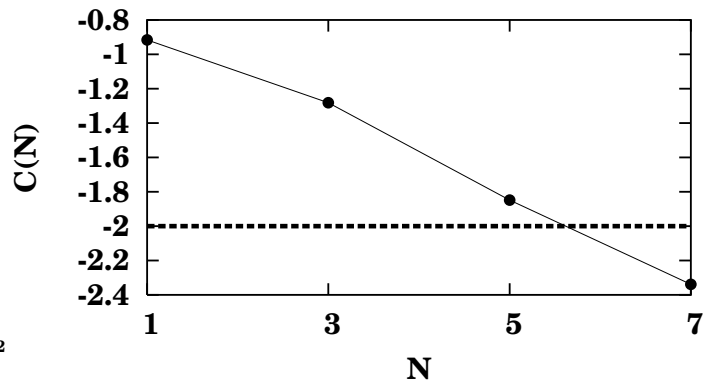

(c)

Figure 4. (a). A slab profile in the cross section of the rectangular straight waveguide. (b). The results between our model and the analytical method. (c). The convergence according to the criterion (14)

According to the proposed technique, the slab dielectric profile $g(x)$ that is shown in Figure 4(a) is given by

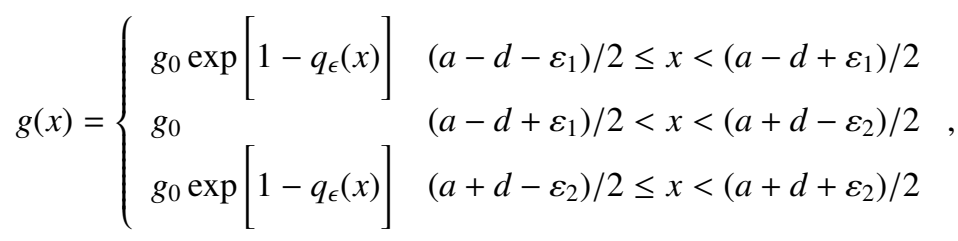

where

$$
q_{\epsilon}(x)=\frac{\varepsilon_{1}^{2}}{\left.\varepsilon_{1}^{2}-\left[x-\left(a-d+\varepsilon_{1}\right) / 2\right)\right]^{2}},
$$

else $g(x)=0$.

The elements of the matrice $g(n, m)$ are given according to Figure $4(\mathrm{a})$ in the case of $\mathrm{b}=\mathrm{c}$ by

$$
g(n, m)=\frac{g_{0}}{a b}\left\{\int_{\left(a-d-\varepsilon_{1}\right) / 2}^{\left(a-d+\varepsilon_{1}\right) / 2} \exp \left[1-q_{\epsilon}(x)\right] \cos \left(\frac{n \pi x}{a}\right) d x+\right.
$$




$$
\left.\int_{\left(a-d-\varepsilon_{1}\right) / 2}^{\left(a+d-\varepsilon_{2}\right) / 2} \cos \left(\frac{n \pi x}{a}\right) d x+\int_{\left(a+d-\varepsilon_{2}\right) / 2}^{\left(a+d+\varepsilon_{2}\right) / 2} \exp \left[1-q_{\epsilon}(x)\right] \cos \left(\frac{n \pi x}{a}\right) d x\right\}\left\{\int_{0}^{b} \cos \left(\frac{m \pi y}{b}\right) d y\right\} .
$$

All the next graphical results are demonstrated as a response to a half-sine $\left(T E_{10}\right)$ input-wave profile and the circular and periodic circular profiles (Figures 1(a)-(d)) in the cross section of the straight rectangular waveguide.

Figures $5(\mathrm{a})$-(d) show the results of the output field as a response to a half-sine $\left(T E_{10}\right)$ input-wave profile. In this case, the radius of the circle is equal to $2.5 \mathrm{~mm}$ and the center of the circle is located at the point $(\mathrm{a} / 2, \mathrm{~b} / 2)$, as shown in Figure 1(a) for $\epsilon_{r}=3,5,7$, and 10, respectively. The other parameters are a $=2 \mathrm{~cm}, \mathrm{z}=15 \mathrm{~cm}, k_{0}=167$ $1 / \mathrm{m}, \lambda=3.75 \mathrm{~cm}$, and $\beta=581 / \mathrm{m}$.

By increasing only the value of the parameter $\epsilon_{r}$ of the circular dielectric profile (Figure 1(a)) in the rectangular cross section from 3 to 10 , the Gaussian shape of the output transverse profile of the field increased and the $T E_{10}$ wave profile decreased. By increasing only the value of the parameter $\epsilon_{r}$, the relative amplitude of the output field decreased.

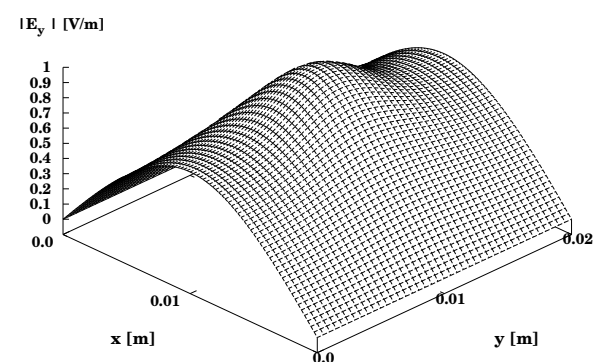

(a)

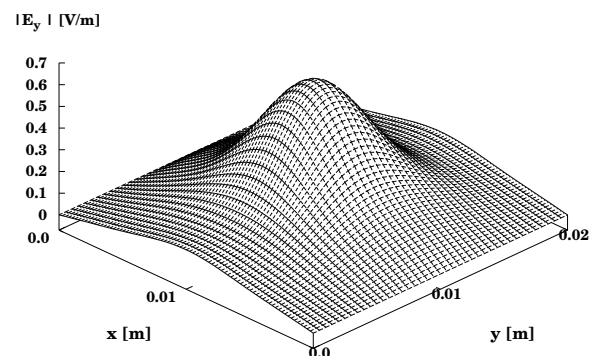

(c)

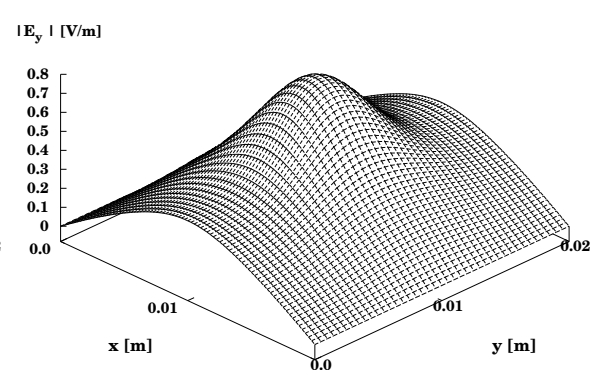

(b)

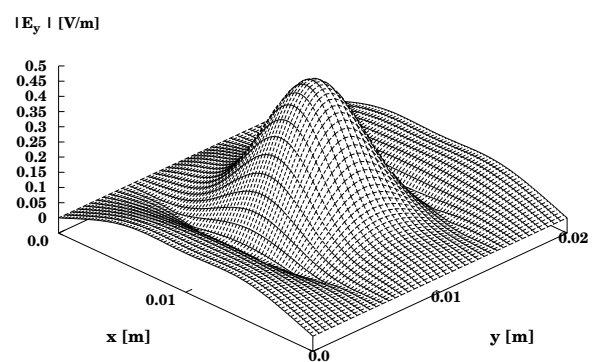

(d)

Figure 5. The output field as a response to a half-sine $\left(T E_{10}\right)$ input-wave profile where the radius of all circle is equal to $2.5 \mathrm{~mm}$ and the center of the circle is located at the point (a/2,b/2) (a). $\epsilon_{r}=3$; (b). $\epsilon_{r}=5 ;$; $) . \epsilon_{r}=7$;

(d). $\epsilon_{r}=10$. The other parameters are $\mathrm{a}=2 \mathrm{~cm}, \mathrm{z}=15 \mathrm{~cm}, k_{0}=1671 / \mathrm{m}, \lambda=3.75 \mathrm{~cm}$, and $\beta=581 / \mathrm{m}$

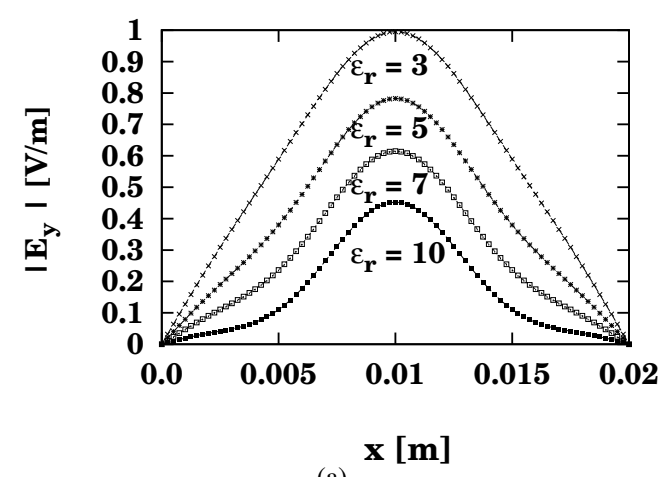

(a)

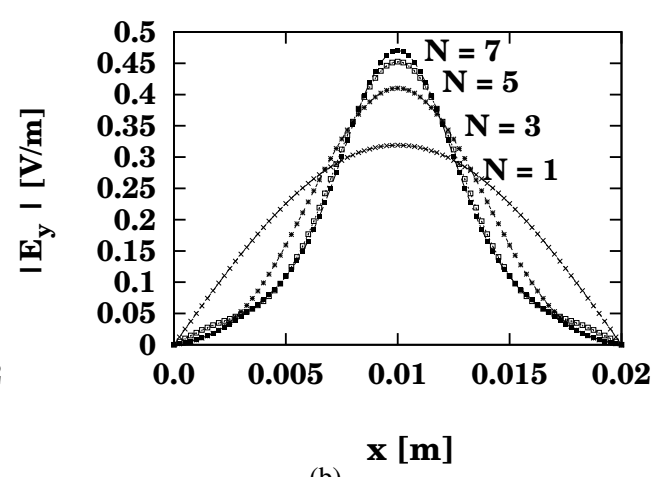

(b)

Figure 6. (a). The output field as a response to a half-sine $\left(T E_{10}\right)$ input-wave profile in the same cross section of Figures 5(a)-(d) for $\epsilon_{r}=3,5,7$, and 10 , respectively, where $\mathrm{a}=\mathrm{b}=20 \mathrm{~mm}, \mathrm{y}=\mathrm{b} / 2=10 \mathrm{~mm}$. The radius of all circle is equal to $2.5 \mathrm{~mm}$ and the center of the circle is located at the point $(\mathrm{a} / 2, \mathrm{~b} / 2)$. (b). The output profiles for

$$
\mathrm{N}=1,3,5 \text {, and } 7 \text {, where } \epsilon_{r}=10
$$


Figure 6(a) shows that by increasing only the value of $\epsilon_{r}$ of the circular dielectric profile, the Gaussian shape of the output field increased, the $T E_{10}$ wave profile decreased, and the relative amplitude decreased. In addition, by increasing only the value of $\epsilon_{r}$, the width of the Gaussian shape decreased. The output profiles for $\mathrm{N}=1,3,5$, and 7 are shown in Figure 6(b) for $\epsilon_{r}=10$. The output field approaches to the final output field, by increasing only the parameter of the order $\mathrm{N}$.

The output fields of Figures 5(a)-(d) and Figures 6(a)-(b) strongly affected by the input wave profile ( $T E_{10}$ mode), the circular profile, and the location of the center of the circle $(a / 2, b / 2)$.

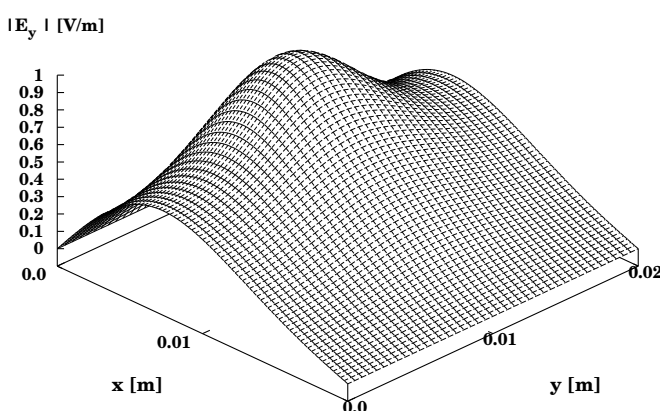

(a)

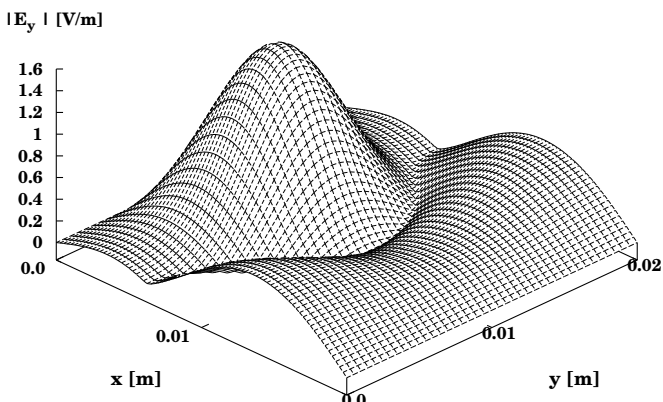

(c)

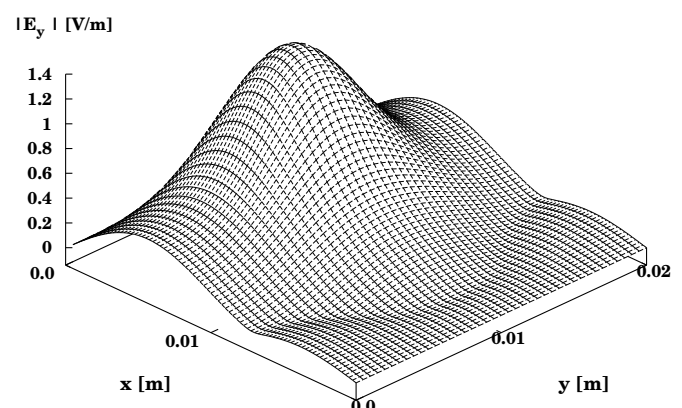

(b)

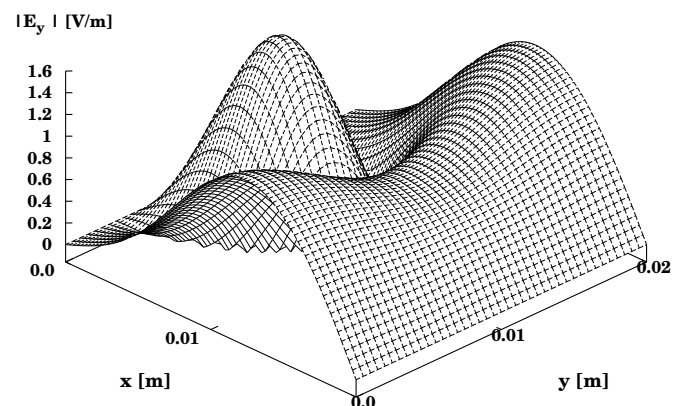

(d)

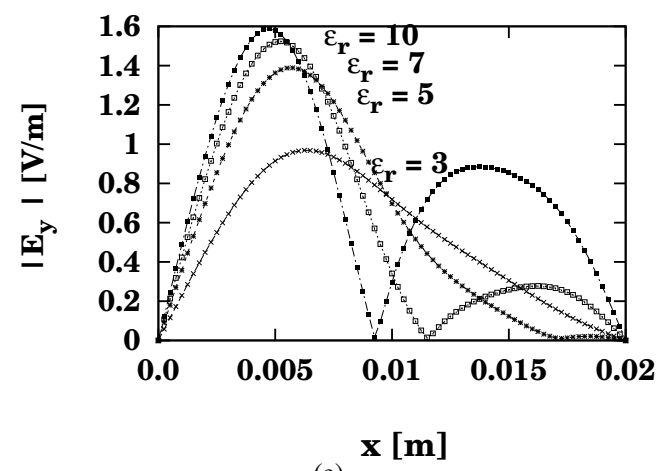

(e)

Figure 7. The output field as a response to a half-sine $\left(T E_{10}\right)$ input-wave profile where the radius of all circle is equal to $2.5 \mathrm{~mm}$ and the center of the circle is located at the point (a/4, b/2) (a). $\epsilon_{r}=3$; (b). $\epsilon_{r}=5 ;$ (c). $\epsilon_{r}=7$; (d). $\epsilon_{r}=10$; (e). The output field in the same cross section of the results (a)-(d) for $\epsilon_{r}=3,5,7$, and 10, respectively,

for $\mathrm{x}$-axis where $\mathrm{y}=\mathrm{b} / 2=10 \mathrm{~mm}$. The other parameters are $\mathrm{a}=2 \mathrm{~cm}, \mathrm{z}=15 \mathrm{~cm}, k_{0}=1671 / \mathrm{m}, \lambda=3.75 \mathrm{~cm}$,

$$
\text { and } \beta=58 \quad 1 / m
$$

Figures 7(a)-(e) show the results of the output field as a response to a half-sine $\left(T E_{10}\right)$ input-wave profile, where the radius of the circle is equal to $2.5 \mathrm{~mm}$ and the center of the circle is located at the point $(\mathrm{a} / 4, \mathrm{~b} / 2)$ for $\epsilon_{r}=3$, 5,7 , and 10, respectively. The other parameters are $\mathrm{a}=2 \mathrm{~cm}, \mathrm{z}=15 \mathrm{~cm}, k_{0}=1671 / \mathrm{m}, \lambda=3.75 \mathrm{~cm}$, and $\beta=58$ $1 / m$. Figure 7(e) shows the output field in the same cross section of the results 7(a)-(d) for $\epsilon_{r}=3,5,7$, and 10 , respectively, and for $\mathrm{x}$-axis where $\mathrm{y}=\mathrm{b} / 2=10 \mathrm{~mm}$.

By changing only the center of the circle from $(\mathrm{a} / 2, \mathrm{~b} / 2)$ to $(\mathrm{a} / 4, \mathrm{~b} / 2)$, the anti-symmetric shapes appear in Figures 
7(a)-(e). By increasing only the parameter $\epsilon_{r}$ from 3 to 10 , the output field is dependent on the input wave profile ( $T E_{10}$ mode) and the Gaussian shape of circular dielectric profile. The output profiles (Figures 7(a)-(d)) are located in the left side with regards to the output profiles (Figures 5(a)-(d)), and the anti-symmetric shapes appear. Figure 7(e) shows that by increasing only the value of $\epsilon_{r}$ of the circular dielectric profile, the anti-symmetric shapes appear the output profiles and the amplitudes increasing.

The output fields (Figures 7(a)-(e)) strongly affected by the input wave profile ( $T E_{10}$ mode), the circular profile, and the location of the center of the circle $(\mathrm{a} / 4, \mathrm{~b} / 2)$.

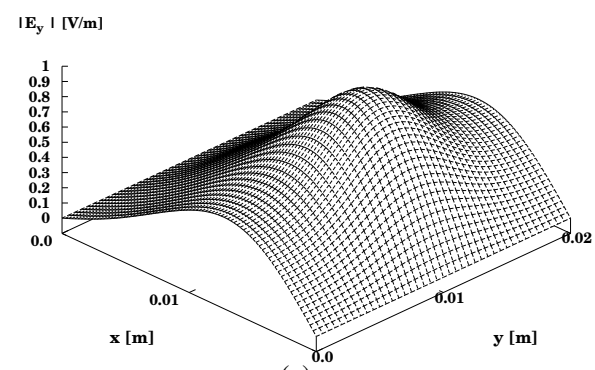

(a)

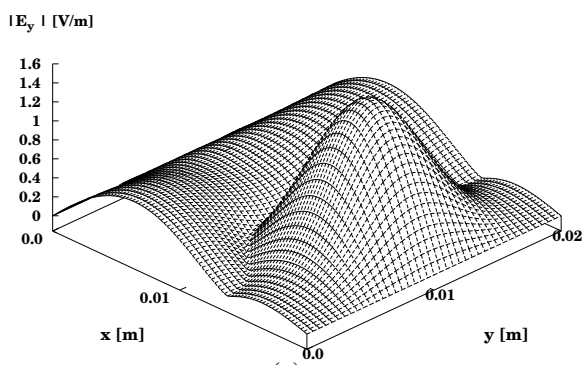

(c)

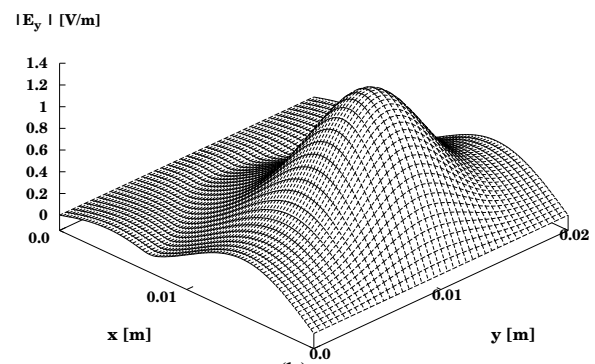

(b)

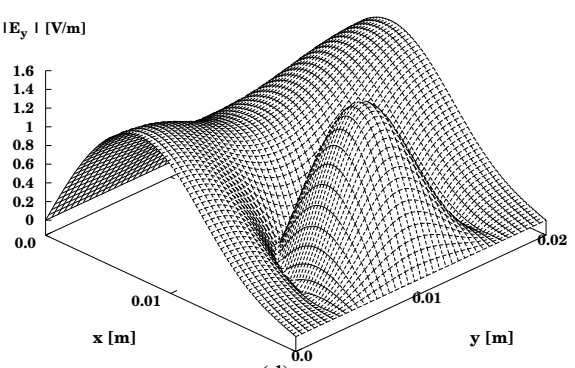

(d)

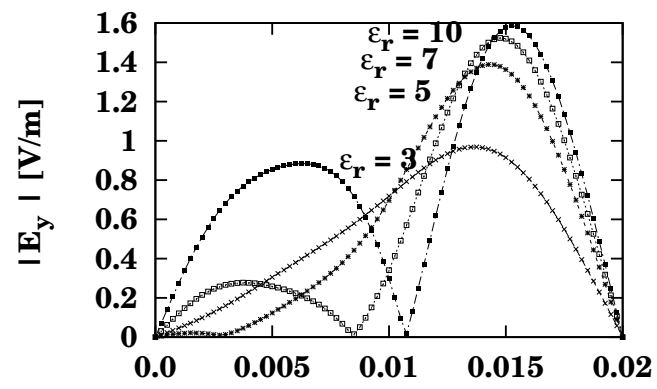

(e)

Figure 8. The output field as a response to a half-sine $\left(T E_{10}\right)$ input-wave profile where the radius of all circle is equal to $2.5 \mathrm{~mm}$ and the center of the circle is located at the point $(3 / 4 \mathrm{a}, \mathrm{b} / 2)(\mathrm{a}) . \epsilon_{r}=3 ;(\mathrm{b}) . \epsilon_{r}=5 ;(\mathrm{c}) . \epsilon_{r}=7$;

(d). $\epsilon_{r}=10$. (e). The output field in the same cross section of the results (a)-(d) for $\epsilon_{r}=3,5,7$, and 10, respectively, for $\mathrm{x}$-axis where $\mathrm{y}=\mathrm{b} / 2=10 \mathrm{~mm}$. The other parameters are $\mathrm{a}=2 \mathrm{~cm}, \mathrm{z}=15 \mathrm{~cm}, k_{0}=1671 / \mathrm{m}$,

$$
\lambda=3.75 \mathrm{~cm} \text {, and } \beta=581 / \mathrm{m}
$$

Figures 8(a)-(e) show the results of the output field as a response to a half-sine $\left(T E_{10}\right)$ input-wave profile, where the radius of the circle is equal to $2.5 \mathrm{~mm}$ and the center of the circle is located at the point $(3 / 4 \mathrm{a}, \mathrm{b} / 2)$ for $\epsilon_{r}=3,5$, 7 , and 10, respectively. The other parameters are $\mathrm{a}=2 \mathrm{~cm}, \mathrm{z}=15 \mathrm{~cm}, k_{0}=1671 / \mathrm{m}, \lambda=3.75 \mathrm{~cm}$, and $\beta=581 / \mathrm{m}$. Figure 8(e) shows the output field in the same cross section of the results of Figures $8(\mathrm{a})$-(d) for $\epsilon_{r}=3,5,7$, and 10 , respectively, and for $\mathrm{x}$-axis where $\mathrm{y}=\mathrm{b} / 2=10 \mathrm{~mm}$.

By changing only the center of the circle from $(\mathrm{a} / 4, \mathrm{~b} / 2)$ to $(3 / 4 \mathrm{a}, \mathrm{b} / 2)$, the anti-symmetric shapes appear in Figures 8(a)-(e). By increasing only the parameter $\epsilon_{r}$ from 3 to 10, the output field is dependent on the input wave profile ( $T E_{10}$ mode) and the Gaussian shape of circular dielectric profile. On the one hand, the output profiles (Figures 8(a)-(e)) are located in the right side with regards to the output profiles (Figures 5(a)-(d)). On the other hand, the 
output profiles (Figures 7(a)-(e)) are located in the left side with regards to the output profiles (Figures 5(a)-(d)). Figure 8(e) shows that by increasing only the value of $\epsilon_{r}$ of the circular dielectric profile, the anti-symmetric shapes appear, the output profiles and the amplitudes increasing. Note that the amplitude of the output field is not changed in the Figures 8(a)-(e) with regards to Figures 7(a)-(e), respectively, for the same value of $\epsilon_{r}$.

The output fields (Figures 8(a)-(e)) strongly affected by the input wave profile ( $T E_{10}$ mode), the circular profile, and the location of the center of the circle $(3 / 4 a, b / 2)$.
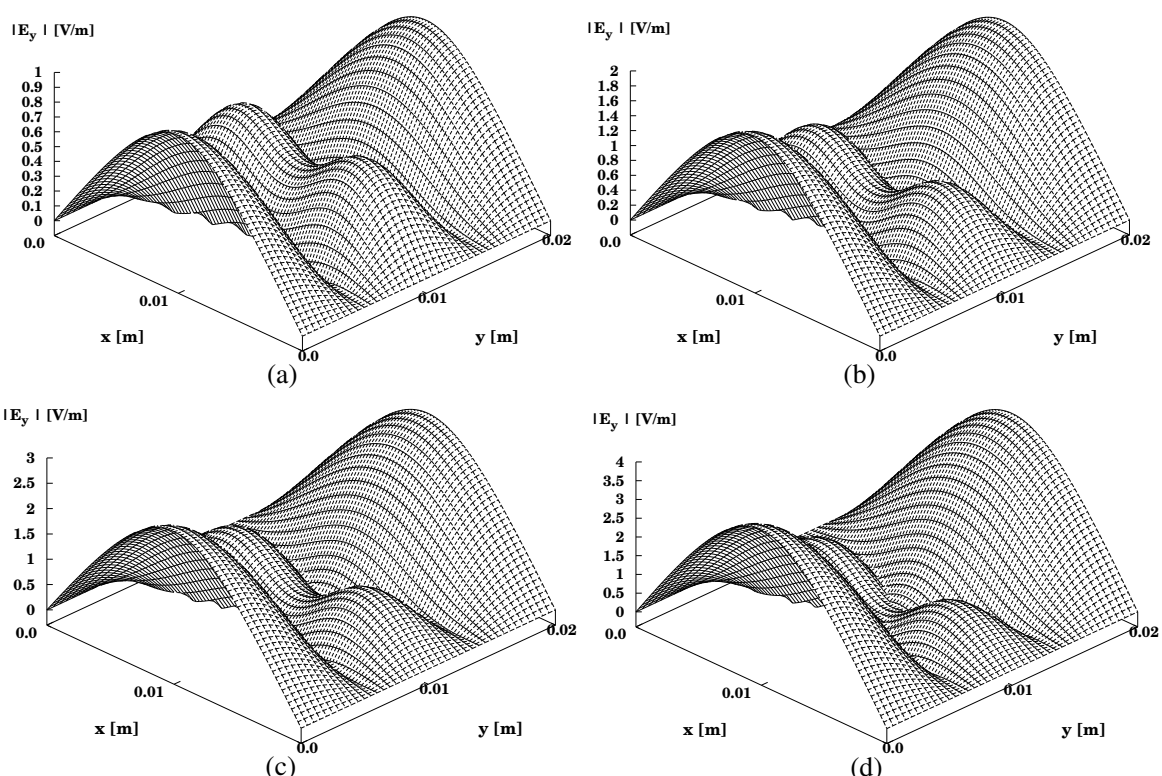

(c)

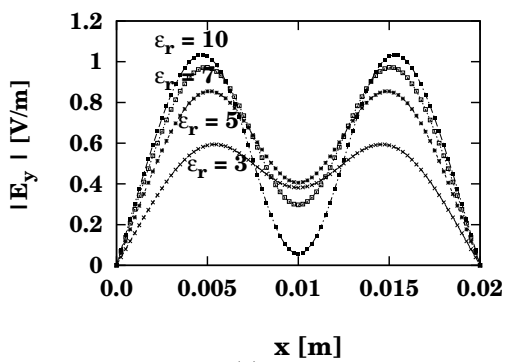

(e)

Figure 9. The output field as a response to a half-sine $\left(T E_{10}\right)$ input-wave profile where the radius of all circle is equal to $1 \mathrm{~mm}$, the center of the left circle is located at the point $(\mathrm{a} / 4, \mathrm{~b} / 2)$ and the center of the right circle is located at the point $(3 / 4 \mathrm{a}, \mathrm{b} / 2)(\mathrm{a}) . \epsilon_{r}=3 ;(\mathrm{b}) . \epsilon_{r}=5 ;$ (c). $\epsilon_{r}=7 ;(\mathrm{d}) . \epsilon_{r}=10$. (e). The output field in the same cross section of the results (a)-(d) for $\epsilon_{r}=3,5,7$, and 10, respectively, for x-axis where $\mathrm{y}=\mathrm{b} / 2=10 \mathrm{~mm}$. The other parameters are $\mathrm{a}=2 \mathrm{~cm}, \mathrm{z}=15 \mathrm{~cm}, k_{0}=1671 / \mathrm{m}, \lambda=3.75 \mathrm{~cm}$, and $\beta=581 / \mathrm{m}$

Figures 9(a)-(e) show the results of the output field as a response to a half-sine $\left(T E_{10}\right)$ input-wave profile, where the radius of the circle is equal to $1 \mathrm{~mm}$, the center of the left circle is located at the point $(\mathrm{a} / 4, \mathrm{~b} / 2)$ and the center of the right circle is located at the point $(3 / 4 \mathrm{a}, \mathrm{b} / 2)$ for $\epsilon_{r}=3,5,7$, and 10 , respectively. The other parameters are $\mathrm{a}=2 \mathrm{~cm}, \mathrm{z}=15 \mathrm{~cm}, k_{0}=1671 / \mathrm{m}, \lambda=3.75 \mathrm{~cm}$, and $\beta=581 / \mathrm{m}$.

In this example, the centers of the left circle and the right circle are located at the points $(\mathrm{a} / 4, \mathrm{~b} / 2)$ and $(3 / 4 \mathrm{a}, \mathrm{b} / 2)$, respectively, as shown in Figure 1(b). Figure 9(e) shows the output field in the same cross section of the results in Figures 9(a)-(d) for $\epsilon_{r}=3,5,7$, and 10, respectively, and for x-axis where $\mathrm{y}=\mathrm{b} / 2=10 \mathrm{~mm}$. In this example, the periodic structure with two circular profilesis shown along $\mathrm{x}$-axis.

The output fields (Figures 9(a)-(e)) strongly affected by the input wave profile ( $T E_{10}$ mode), the periodic structure with the two circular profiles along x-axis (Figure 1(b)), and the distance between the two centers of the circular profiles. 


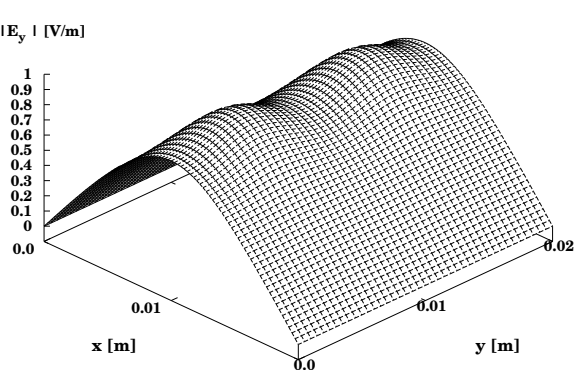

(a)

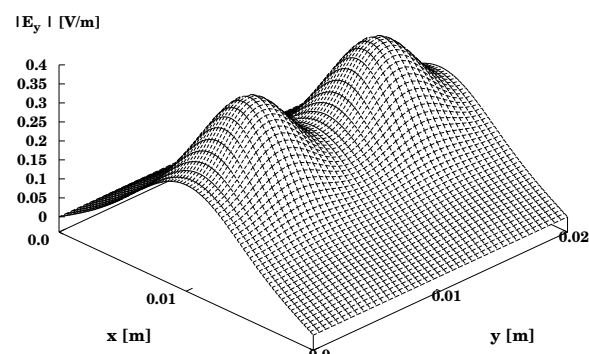

(c)

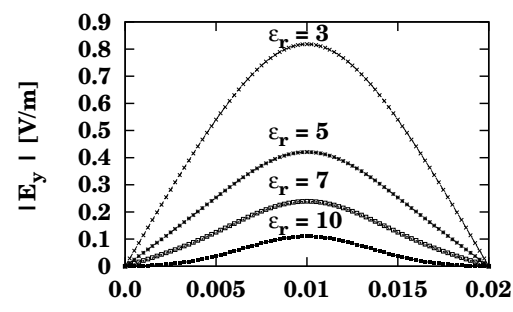

(e)

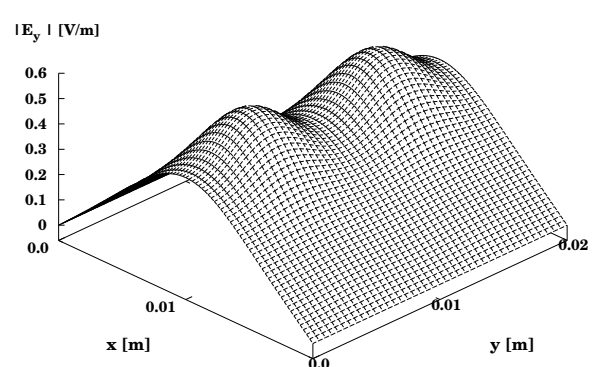

(b)

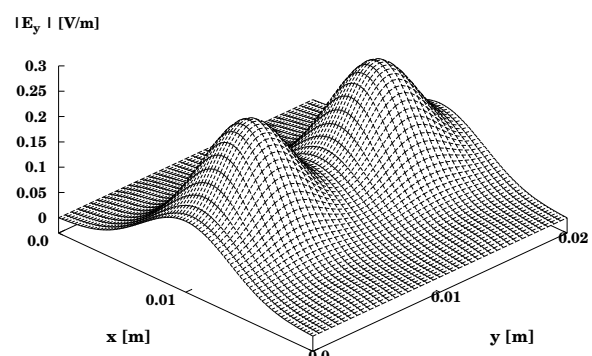

(d)

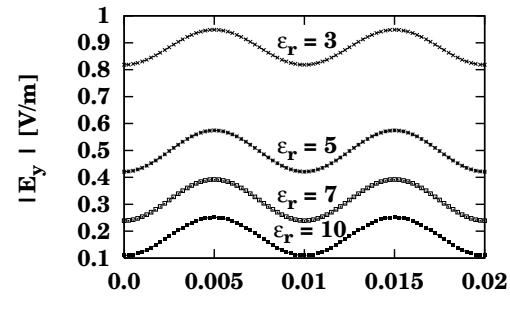

(f)

Figure 10. The output field as a response to a half-sine $\left(T E_{10}\right)$ input-wave profile where the radius of all circle is equal to $2.5 \mathrm{~mm}$, the center of the upper circle is located at the point $(\mathrm{a} / 2,3 / 4 \mathrm{~b})$ and the center of the lower circle is located at the point (a/2 a, b/4) for (a). $\epsilon_{r}=3$; (b). $\epsilon_{r}=5 ;$ (c). $\epsilon_{r}=7$; (d). $\epsilon_{r}=10$. The output field in the same cross section of the same results (a)-(d) for $\epsilon_{r}=3,5,7$, and 10, respectively, for two cases: (e). for x-axis where $\mathrm{y}=\mathrm{b} / 2=10 \mathrm{~mm}$. (f). for $\mathrm{y}$-axis where $\mathrm{x}=\mathrm{a} / 2=10 \mathrm{~mm}$. The other parameters are $\mathrm{a}=2 \mathrm{~cm}, \mathrm{z}=15 \mathrm{~cm}, \mathrm{k}_{0}=1671 / \mathrm{m}$,

$$
\lambda=3.75 \mathrm{~cm} \text { and } \beta=581 / \mathrm{m}
$$

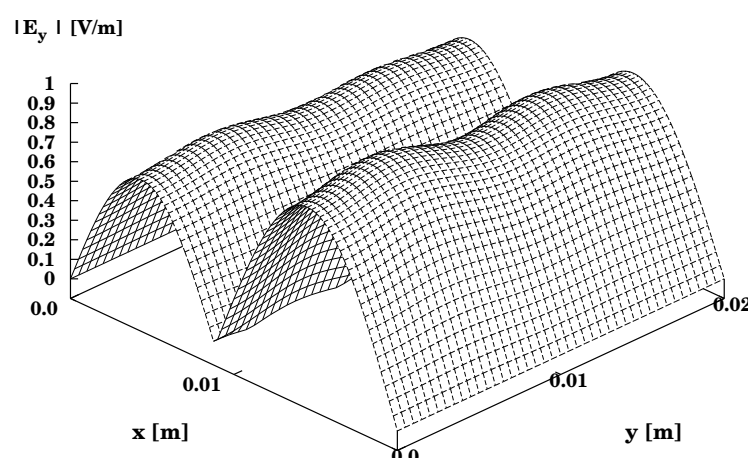

(a)

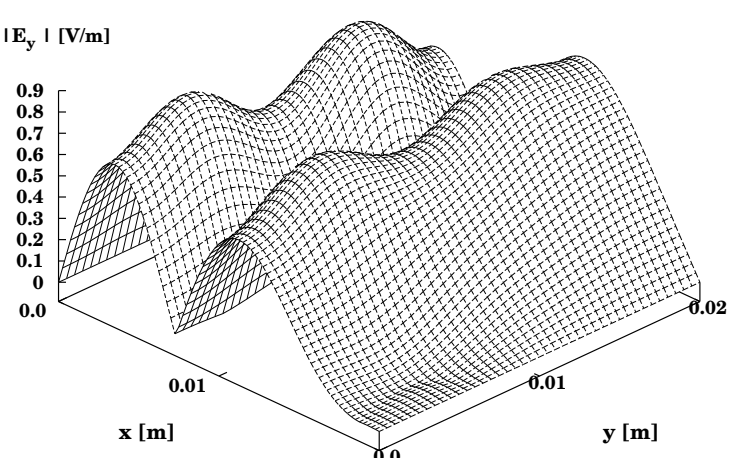

(b)

Figure 11. The output field as a response to a half-sine $\left(T E_{10}\right)$ input-wave profile where the radius of all circle is equal to $5 \mathrm{~mm}$, the center of the first circle is located at the point $(\mathrm{a} / 4, \mathrm{~b} / 4)$, the center of the second circle is located at the point $(3 / 4 \mathrm{a}, \mathrm{b} / 4)$, the center of the third circle is located at the point $(\mathrm{a} / 4,3 / 4 \mathrm{~b})$, and the center of the firth circle is located at the point $(3 / 4 \mathrm{a}, 3 / 4 \mathrm{~b})$. (a). $\epsilon_{r}=5 ;$ (b). $\epsilon_{r}=10$. The other parameters are a $=2 \mathrm{~cm}$,

$$
\mathrm{z}=15 \mathrm{~cm}, k_{0}=1671 / \mathrm{m}, \lambda=3.75 \mathrm{~cm} \text { and } \beta=581 / \mathrm{m}
$$


Figures 10(a)-(f) show the results of the output field as a response to a half-sine (TE $\left.E_{10}\right)$ input-wave profile for $\epsilon_{r}$ $=3,5,7$, and 10, respectively. In this example, the radius of the circle is equal to $2.5 \mathrm{~mm}$, the centers of the upper circle and the lower circle are located at the points $(\mathrm{a} / 2,3 / 4 \mathrm{~b})$ and $(\mathrm{a} / 2, \mathrm{~b} / 4)$, respectively, as shown in Figure 1(c). The other parameters are $\mathrm{a}=2 \mathrm{~cm}, \mathrm{z}=15 \mathrm{~cm}, k_{0}=1671 / \mathrm{m}, \lambda=3.75 \mathrm{~cm}$, and $\beta=581 / \mathrm{m}$.

The output fields (Figures 10(a)-(f)) strongly affected by the input wave profile ( $T E_{10}$ mode), the periodic structure with the two circular profiles along y-axis (Figure 1(c)), and the distance between the two centers of the circular profiles.

The output fields in the same cross section of the results of Figures 10(a)-(d) are shown in Figure 10(e) for xaxis where $\mathrm{y}=\mathrm{b} / 2=10 \mathrm{~mm}$ and are shown in Figure 10(f) for $\mathrm{y}$-axis where $\mathrm{x}=\mathrm{a} / 2=10 \mathrm{~mm}$ for $\epsilon_{r}=3,5,7$, and 10 , respectively. By changing only the parameter $\epsilon_{r}$ from 3 to 10 , the relative profile of the output field is changed from a half-sine $\left(T E_{10}\right)$ profile to a Gaussian shape profile, as shown in Figure 10(e). The results of the periodic structures of the output field along $\mathrm{x}$-axis and $\mathrm{y}$-axis are demonstrated in Figures 11(a)-(b). These results strongly affected by the half-sine $\left(T E_{10}\right)$ input-wave profile, the locations of the circular profiles along $\mathrm{x}$-axis and along $y$-axis (Figure 1(d)), and the distance between the fourth centers of the circular profiles.

Figures 11(a)-(b) show the results of the output field as a response to a half-sine ( $\left.T E_{10}\right)$ input-wave profile, where the radius of all circle is equal to $5 \mathrm{~mm}$, the center of the first circle is located at the point $(\mathrm{a} / 4, \mathrm{~b} / 4)$, the center of the second circle is located at the point $(3 / 4 \mathrm{a}, \mathrm{b} / 4)$, the center of the third circle is located at the point $(\mathrm{a} / 4,3 / 4$ b), and the circle of the firth circle is located at the point ( $3 / 4 \mathrm{a}, 3 / 4 \mathrm{~b})$ for $\epsilon_{r}=5$, and 10 , respectively. The other parameters are $\mathrm{a}=2 \mathrm{~cm}, \mathrm{z}=15 \mathrm{~cm}, k_{0}=1671 / \mathrm{m}, \lambda=3.75 \mathrm{~cm}$, and $\beta=581 / \mathrm{m}$.

The results of the periodic structures of the output field along $\mathrm{x}$-axis and $\mathrm{y}$-axis are demonstrated in Figures 11(a)(b). These results strongly affected by the half-sine $\left(T E_{10}\right)$ input-wave profile, the locations of the circular profiles along $\mathrm{x}$-axis and along $\mathrm{y}$-axis (Figure 1(d)), and the distance between the fourth centers of the circular profiles. By increasing the parameter $\epsilon_{r}$ from 5 to 10, the Gaussian shape of the output field increased.

\section{Conclusions}

The first objective of this paper was to present a technique and a particular application to calculate the dielectric profile, the elements of the matrix, and its derivatives of the dielectric profile in the cases of circular and periodic circular profiles in the cross section of the rectangular straight waveguide. The second objective was to investigate the influence of the circular and periodic circular profiles on the output fields.

The technique was proposed in detail in order to understand how to calculate the elements of the matrix and its derivatives. The output results were demonstrated for circular profile and for periodic circular profiles in the cross section.

A comparison with the known transcendental equation was shown in order to examine the validity of the theoretical model, by using with the proposed technique. The result of the comparison between the theoretical model with the known solution (Collin, 1996) has shown good agreement.

All the graphical results were demonstrated as a response to a half-sine $\left(T E_{10}\right)$ input-wave profile and the circular and periodic circular profiles (Figures 1(a)-(d)) in the cross section of the straight rectangular waveguide. The calculation of the elements of the matrix $\mathrm{g}(\mathrm{n}, \mathrm{m})$ for an arbitrary profile in the cross section of the straight waveguide is given according to Figure 2 .

The results of the output field as a response to a half-sine $\left(T E_{10}\right)$ input-wave profile show that by increasing only the value of the parameter $\epsilon_{r}$ of the circular dielectric profile (Figure 1(a)) in the rectangular cross section from 3 to 10, the Gaussian shape of the output transverse profile of the field increased, the $T E_{10}$ wave profile decreased, and the width of the Gaussian shape decreased.

By changing only the center of the circle from $(a / 2, b / 2)$ to $(a / 4, b / 2)$, or by changing only the center of the circle from $(a / 4, b / 2)$ to $(3 / 4 a, b / 2)$, the anti-symmetric shapes appear, the output profiles and the amplitudes increasing.

The results of the output fields strongly affected by the input wave profile ( $T E_{10}$ mode), the circular profile, and the location of the center of the circle.

This model is useful to predict the structure of the output fields for circular and periodic circular profiles in a rectangular metallic waveguide. The application is useful for straight waveguides in the microwave and the millimeterwave regimes. 


\section{Appendix A}

The dielectric profile $g(x, y)$ is given according to $\epsilon(x, y)=\epsilon_{0}(1+g(x, y))$. According to Figure 2 and for $g(x, y)=g_{0}$, we obtain

$$
\begin{gathered}
g(n, m)=\frac{g_{0}}{4 a b} \int_{-a}^{a} d x \int_{-b}^{b} \exp \left[-j\left(k_{x} x+k_{y} y\right)\right] d y \\
=\frac{g_{0}}{4 a b}\left\{\int_{x_{11}}^{x_{12}} d x \int_{y_{11}}^{y_{12}} \exp \left[-j\left(k_{x} x+k_{y} y\right)\right] d y+\int_{-x_{12}}^{-x_{11}} d x \int_{y_{11}}^{y_{12}} \exp \left[-j\left(k_{x} x+k_{y} y\right)\right] d y\right. \\
\left.+\int_{-x_{12}}^{-x_{11}} d x \int_{-y_{12}}^{-y_{11}} \exp \left[-j\left(k_{x} x+k_{y} y\right)\right] d y+\int_{x_{11}}^{x_{12}} d x \int_{-y_{12}}^{y_{11}} \exp \left[-j\left(k_{x} x+k_{y} y\right)\right] d y\right\} \\
=\frac{g_{0}}{4 a b}\left\{\int_{x_{11}}^{x_{12}} d x \int_{y_{11}}^{y_{12}} \exp \left[-j\left(k_{x} x+k_{y} y\right)\right] d y+\int_{x_{12}}^{x_{11}}-d x \int_{y_{11}}^{y_{12}} \exp \left[-j\left(-k_{x} x+k_{y} y\right)\right] d y\right. \\
\left.+\int_{x_{12}}^{x_{11}}-d x \int_{y_{12}}^{y_{11}}-\exp \left[-j\left(-k_{x} x-k_{y} y\right)\right] d y+\int_{x_{11}}^{x_{12}} d x \int_{y_{12}}^{y_{11}}-\exp \left[-j\left(k_{x} x-k_{y} y\right)\right] d y\right\} \\
=\frac{g_{0}}{4 a b}\left\{\int_{x_{11}}^{x_{12}} \exp \left[j\left(k_{x} x\right)\right] d x \int_{y_{11}}^{y_{12}} \exp \left[j\left(k_{y} y\right)\right] d y+\int_{x_{11}}^{x_{12}} \exp \left[j\left(k_{x} x\right)\right] d x \int_{y_{11}}^{y_{12}} \exp \left[-j\left(k_{y} y\right)\right] d y\right. \\
\left.+\int_{x_{11}}^{x_{12}} \exp \left[-j\left(k_{x} x\right)\right] d x \int_{y_{11}}^{y_{12}} \exp \left[j\left(k_{y} y\right)\right] d y+\int_{x_{11}}^{x_{12}} \exp \left[-j\left(k_{x} x\right)\right] d x \int_{y_{11}}^{y_{12}} \exp \left[-j\left(k_{y} y\right)\right] d y\right\} \\
\quad=\frac{g_{0}}{4 a b}\left\{\int_{x_{11}}^{x_{12}} d x \int_{y_{11}}^{y_{12}} \exp \left[-j\left(k_{x} x+k_{y} y\right)\right] d y+\int_{x_{11}}^{x_{12}} d x \int_{y_{11}}^{y_{12}} \exp \left[j\left(k_{x} x-k_{y} y\right)\right] d y\right. \\
\left.+\int_{x_{11}}^{x_{12}} d x \int_{y_{11}}^{y_{12}} \exp \left[j\left(k_{x} x+k_{y} y\right)\right] d y+\int_{x_{11}}^{x_{12}} d x \int_{y_{11}}^{y_{12}} \exp \left[-j\left(k_{x} x-k_{y} y\right)\right] d y\right\} \\
\left.g_{0} a b \int_{x_{11}}^{x_{12}}\left(\exp \left(j k_{x} x\right)+\exp \left(-j k_{x} x\right)\right) d x \int_{y_{11}}^{y_{12}} \cos \left(k_{y} y\right) d y\right\}
\end{gathered}
$$

\section{References}

Abbas, Z., Pollard, R. D. \& Kelsall, W., (1998). A rectangular dielectric waveguide technique for determination of permittivity of materials at W-band. IEEE Transactions on Microwave Theory and Techniques, 46, 2011-2015. http://dx.doi.org/10.1109/22.739275

Choocadee, S., \& Akatimagool, S. (2012). The simulation, design and implementation of bandpass filters in rectangular waveguides. Electrical and Electronic Engineering, 2, 152-157. http://dx.doi.org/10.5923/j.eee.201202 03.08

Collin, R. E. (1996). Foundation for Microwave engineering. New York: McGraw-Hill.

Eyges, L., \& Gianino, P. (1979). Modes of dielectric waveguides of arbitrary cross sectional shape. Journal Opt. Soc. Am., 69, 1226-1235. http://dx.doi.org/10.1364/JOSA.69.001226

Hewlett, S. J., \& Ladouceur, F. (1995). Fourier decomposition method applied to mapped infinite domains: scalar analysis of dielectric waveguides down to modal cutoff. Journal of Lightwave Technology, 13, 375-383. http://dx.doi.org/10.1109/50.372431

Jiao, C. Q. (2011). Selective suppression of electromagnetic modes in a rectangular waveguide by using distributed wall losses. Progress In Electromagnetics Research Letters, 22, 119-128.

Liu, S., Li, L. W., Leong, M. S., \& Yeo, T. S. (2000). Rectangular conducting waveguide filled with uniaxial anisotropic media: a modal analysis and dyadic Green's function. Progress In Electromagnetics Research, 25, 111-129. http://dx.doi.org/10.2528/PIER99052501 
Lu, W., \& Lu, Y. Y. (2012). Waveguide mode solver based on Neumann-to-Dirichlet operators and boundary integral equations. Journal of Computational Physics, 231, 1360-1371. http://dx.doi.org/10.1016/j.jcp.2011.10.016

Menachem, Z., \& Jerby, E. (1998). Transfer Matrix Function (TMF) for propagation in dielectric waveguides with arbitrary transverse profiles. IEEE Transactions On Microwave Theory and Techniques, 46, 975-982. http://dx.doi.org/10.1109/22.701451

Menachem, Z., \& Tapuchi, S. (2013). Influence of the spot-size and cross-section on the output fields and power density along the straight hollow waveguide. Progress In Electromagnetics Research, 48, 151-173. http://dx. doi.org/10.2528/PIERB12112009

Mineo, M., Carlo, A. Di., \& Paoloni, C. (2010). Analytical design method for corrugated rectangular waveguide SWS THZ vacuum tubes. Journal of Electromagnetic Waves and Applications, 24, 2479-2494. http://dx.doi. org/10.1163/156939310793675745

Rothwell, E. J., Temme, A., \& Crowgey, B. (2009). Pulse reflection from a dielectric discontinuity in a rectangular waveguide. Progress In Electromagnetics Research, 97, 11-25. http://dx.doi.org/10.2528/PIER09090905

Salzer, H. E. (1955). Orthogonal polynomials arising in the numerical evaluation of inverse Laplace transforms. Math. Tables and Other Aids to Comut., 9, 164-177. http://dx.doi.org/10.2307/2002053

Salzer, H. E. (1961). Additional formulas and tables for orthogonal polynomials originating from inversion integrals. J. Math. Phys., 39, 72-86.

Sharma, J. (2010). Full-wave analysis of dielectric rectangular waveguides. Progress In Electromagnetics Research, 13, 121-131. http://dx.doi.org/10.2528/PIERM10051802

Soekmadji, H., Liao, S. L., \& Vernon, R. J. (2009). Experiment and simulation on $T E_{10}$ cut-off reflection phase in gentle rectangular downtapers. Progress In Electromagnetics Research Letters, 12, 79-85. http://dx.doi.org/10. 2528/PIERL09090707

Vladimirov, V. S., Jeffrey, A., \& Littlewood, A. (1971). Equations of Mathematical Physics (pp. 66-67). New York: M. Dekker.

Yeap, K. H., Tham, C. Y., Yassin, G., \& Yeong, K. C. (2011). Attenuation in rectangular waveguides with finite conductivity walls. Radioengineering, 20, 472-478.

\section{Copyrights}

Copyright for this article is retained by the author(s), with first publication rights granted to the journal.

This is an open-access article distributed under the terms and conditions of the Creative Commons Attribution license (http://creativecommons.org/licenses/by/3.0/). 